\title{
Molecular Phylogenetic Analysis of Vicia L. (Fabaceae) Taxa Growing in the Southeastern Anatolia Region of Turkey: Based on Internal Transcribed Spacer (ITS)
}

\author{
Alevcan Kaplan ${ }^{1, a, *}$, A. Selçuk Ertekin ${ }^{2, b}$, Esra Gündüzer ${ }^{3, c}$ \\ ${ }^{1}$ Sason Vocational School, Batman University, 72060 Batman, Turkey \\ ${ }^{2}$ Ekinciler Street, Kalender Center, Floor: 6, Apart. No: 9- 21120-Yenişehir/Diyarbakır, Turkey \\ ${ }^{3}$ Life Sciences Application and Research Center, Gazi University, 06500 Ankara, Turkey
}

*Corresponding author

\section{A R T I C L E IN F O A B S T R A C T}

Research Article

Leguminosae or Fabaceae is the third-largest flowering plant family and is important in terms of both food production and soil fertility. Wild Vicia species and the genetic diversity of the Southeastern Anatolia Region provide an invaluable resource for the improvement of cultivated temperate feed and legume crops. The rapid progress of technology in recent years has nowmade it possible to use modern techniques in phylogenetic studies and to examine plants in a greater detail using biochemical, cytological and molecular methods to supplement purely systematic studies. The use of molecular phylogenetic analysis is the most attractive alternative strategy for a more accurate identification of the species of the Vicia genus. In the current study, some Vicia L. taxa growing naturally in the Southeastern Anatolia Region were investigated using molecular phylogenetic analysis. Internal transcribed spacers (ITS) of nuclear ribosomal DNA were sequenced in order to

Keywords:

ITS

Fabaceae

Molecular phylogeny

nrDNA

Vicia study the phylogenetic relationships of Vicia L. taxa. Lathyrus inconspicuous L. and Lathyrus cassius Boiss. were used as an outgroup. The ITS area was determined to be approximately 479$672 \mathrm{bp}$. The ITS sequences were submitted to the NCBI database and accession numbers obtained. The resulting tree clearly groups and separates the sect. Narbonensis, Ervilia, Peregrinae, Lathyroides, Vicia and Cracca species but was less able to distinguish species from sect. Hypechusa and Lentopsis. The data acquired were observed to be reliable in terms of solving the taxonomical problems of the Vicia L. taxa. The morphological distinctions are greatly supported by DNA sequence studies. The species-specific markers developed in this study are useful for early detection of targeted Vicia taxa and can act as a guide to the basic data required for the evolution of systematic breeding and conservation strategies, as well as for germplasm resources.

\section{(c) (1) T) This work is licensed under Creative Commons Attribution 4.0 International License}

\section{Introduction}

Fabaceae (Leguminosae) is the third largest flowering plant family, comprising over 765 genera and $\sim 19500$ species (LPWG, 2017; Legume Phylogeny Working Group [LPWG]). The family is generally divided into three subfamilies, Caesalpinioideae (Paraphyletic), Mimosoideae and Papilionoideae (Monophyletic) (Davis, 1970; Heywood et al., 2007; Miller et al., 2011; Simpson, 2012).

The economically important and widespread genus Vicia L. comprises annual or perennial herbaceous species spread throughout the temperate regions of Europe, Asia, and North and South America. The number of species recognized in the genus varies significantly and is estimated to be between 150 and 210 (Kupicha, 1976; Hanelt and Mettin, 1989). Vavilov (1994) discussed how Turkey contains many of the diversity and gene centers of the genus in the Mediterranean and Near East. In particular,
Eastern Anatolia and some parts of southeastern Anatolia have the third-largest number of genetically differentiated examples of Vicia L. species in the world (Vavilov, 1950). 66 species, 22 subspecies and 29 varieties have been reported in Turkey. 11 of these taxa were endemic (Başbağ et al., 2013). After Russia, Turkey has the second most abundant growth of Vicia L. species (Kaplan, 2014).

It has been reported that there are a number of taxonomic problems with the Vicia genus, and there is as yet no monograph on this genus (Davis, 1970). As with many variable cultivated plants, there is great confusion about the taxonomy and nomenclature of Vicia species (Guidetta and Michelozzi, 1986). Despite the fact that the morphological characters, anatomy, and ecological and karyological (Tabur et al., 2002; Navratilova et al., 2003; Bryant and Hughes, 2011; Kahraman et al., 2013) features of Vicia genus have 
all been studied in order to try and resolve such problems, the number of molecular studies is still not sufficient. Generally, diagnoses of the major subgeneric groupings within the subgenus Vicia have relied heavily on a few diagnostic characters that have been used commonly and consistently. These are: the presence of peduncle and, if present, the length of the peduncle in relation to either the flower or the subtending leaf length; the number of flowers per inflorescence; the presence of stipular nectarines; and the positioning of hairs around the style. Many more characters are used, however, to distinguish subgroups in the subgenus (Maxted, 1995). These characters are life span, plant stature, the number of flowers per inflorescence, the number of leaflets per leaf, the size of leaflets, calyx mouth shape, flower color, vexillum shape, vexillum pubescence, legume pubescence, legume protrusion, relative legume size, relative legume shape, legume suture curvature, legume hair types, seed shape, relative hilum length, relative position of the seed hilum and lens, and seed surface type. A key to Vicia subgenus Vicia sections, series, and species was presented by Maxted (1995). Hosseinzadeh et al. (2008) reported, however, that there are several specimens with intermediate morphological characters and that there are many polymorphisms in these characters that share a common habitat. This means that species diagnosis using morphological characters is a difficult task for nonspecialists and results are not always accurate (Haider et al., 2010). However, the rapid advancement of technology has provided the opportunity to use more modern methods, and taxa have begun to be evaluated using molecular techniques rather than purely systematic studies. Because none of the previous classifications completely reflects the phylogenetic relationship of flowering plants researchers have turned to DNA sequence studies and other modern studies (Felstein, 1985). Recently, the phylogenetic relationships of Vicia species have been studied using nucleotide sequences of different regions of DNA (Haider et al., 2012; Foladi et al., 2013; Saqer, 2014). This study was thus carried out to understand the evolutionary relationships of the taxa belonging to Vicia genus which have spread naturally in the Southeastern Anatolia Region and to delimit the borders of the species.

We chose to use the internal transcribed spacer (ITS; ITS1 intergenic spacer+5.8S gene+ITS2 intergenic spacer) region of nuclear DNA. ITS of $18 \mathrm{~S}-26 \mathrm{~S}$ nuclear ribosomal DNA (nrDNA) is suitable for molecular systematic studies (Aceto et al., 1999) due to its variability at the species level. The main objectives of the current study were: i) to shed further light on the systematics and evolutionary structure of Vicia taxa living in Southeastern Anatolia Region by using the sequence diversity of the regions of ITS; ii) to work out the phylogenetic relationships among sections and subsections including native species; iii) to understand the usefulness of the regions for the phylogeny of Vicia genus.

The Fabaceae (Legumes) family, which contains important cultivars found inlarge parts of the world, is also very important for Turkey, which generally has an agricultural economy. Identifying the variations between species in this genus and resolving the taxonomic problems are very important tasks in terms of their economic contribution to future breeding studies.

\section{Material and Methods}

\section{Plant Material}

Plant material was collected from different localities in the study area from April to May, which is the vegetation periods of the plants in natural habitats, and was identified according to the diagnostic morphological characteristics described in the Flora of Turkey and the East Aegean Islands (Davis, 1970) (Table 1). The study area is located in the Southeastern Anatolia Region (the provinces of Adiyaman, Batman, Diyarbakır, Gaziantep, Mardin, Siirt, Şanlıurfa). Species were included in eight different sections; Cracca S.F. Gray, Ervilia (DC) Link, Lentopsis Kupicha, Peregrinae Kupicha, Lathyroides (Buchenau) Tzvel, Hypechusa (Alef.) Aschers \& Graebner, Vicia L. and Narbonensis (Radzhi) Maxted. To construct the phylogenetic relationships of the Vicia genus, we analyzed sequences of ITS regions from a total of 22 specimens and 25 taxa. Lathyrus cassius Boiss. and Lathyrus inconspicuous L. were used as an outgroup. DNA sequences of the species studied were entered into the NCBI database and an accession number was obtained for each species and listed in Table 1. Plants were deposited under suitable conditions at the Herbarium of the Department of Biology (DUF), Faculty of Science and Art, Dicle University.

\section{Genomic DNA extraction}

Using the Lefort et al. (1998) method total genomic DNA was isolated from leaf tissues that had been dried and stored at room temperature. The purity and quantity of the extracted DNA were determined using the NanoDrop 1000c UV-Vis Spectrophotometer (Thermo Scientific) at $\mathrm{A}_{260} / \mathrm{A}_{280} \mathrm{~nm}$. The isolated DNA samples were checked in $1 \%$ agarose gel electrophoresis (Figure 1).

\section{PCR Analysis and Agarose Gel Electrophoresis}

Specific primer pairs were used to amplify the targeted region on genomic DNA. Amplification of the ITS region was carried out by using primer pairs ITS1 5, TCC GTA GGT GAA CCT GCG G (forward) / ITS4 5' TCC TCC GCT TAT TGA TAT GC 3' (reverse) (White et al., 1990). DNA amplification was performed in a $50 \mu \mathrm{L}$ volume mixture containing genomic DNA (100 ng/ $\mu \mathrm{L}), 10 \mathrm{X}$ PCR Buffer, $\mathrm{MgCl}_{2}(25 \mathrm{mM})$, dNTP mixture (25 mM), selected primer pair $(50 \mu \mathrm{M})$, Taq polymerase $(5 \mathrm{u} / \mu \mathrm{L})$ and sterile water $\left(\mathrm{ddH}_{2} \mathrm{O}\right)$. PCR reaction of each region consisted of almost same amount of Buffer $(5 \mu \mathrm{L})$, DNA $(1 \mu \mathrm{L}), \mathrm{MgCl}_{2}(1.5 \mu \mathrm{L})$, dNTP $(1 \mu \mathrm{L})$, each primer (forward and reverse) $(1.5 \mu \mathrm{L})$ and Taq polymerase $(0.3 \mu \mathrm{L})$. PCR amplification was started with 2 min initial denaturation at $94^{\circ} \mathrm{C}$, and terminated with $5 \mathrm{~min}$ at $72^{\circ} \mathrm{C}$. Each reaction ended with a final $4^{\circ} \mathrm{C}$ hold step and consisted of 35 cycle numbers. Each reaction cycle consisted of a denaturation step at $94^{\circ} \mathrm{C}$ for $20 \mathrm{sec}(1 \mathrm{~min})$, an annealing step at $58^{\circ} \mathrm{C}$ for $30 \mathrm{sec}$ and an elongation step at $72^{\circ} \mathrm{C}$ for 30 $\sec (1 \mathrm{~min})$. Amplicons were visualized by electrophoresis on $2 \%$ agarose gel (Figure 2). After purification, the products were sequenced in both directions using an Applied 3130 Genetic Analyzer automated sequencer. All sequence chromatograms were opened using DNA dragon program. Ambiguous sites were checked manually and corrected by comparing the strands. Sequences of ITS regions were deposited in NCBI database for further studies (accession numbers were indicated in Table 1). 
Table 1. The taxa of Vicia L. studied and their collecting sites, section, accession number and voucher name and herbarium number

\begin{tabular}{|c|c|c|c|c|c|}
\hline No & Taxon names & Section & Location & $\begin{array}{l}\text { Accession } \\
\text { number }\end{array}$ & $\begin{array}{l}\text { Voucher name and } \\
\text { herbarium no }\end{array}$ \\
\hline 1 & $\begin{array}{l}\text { V.narbonensis L.var. } \\
\text { narbonensis }\end{array}$ & Narbonensis & $\begin{array}{l}\text { Batman, Batman University, } \\
\text { central campus, } 570 \mathrm{~m}\end{array}$ & MN733368 & A.Kaplan $D U F 90$ \\
\hline 2 & V. hybrida $\mathrm{L}$. & Hypechusa & $\begin{array}{l}\text { Batman, Batman University, } \\
\text { central campus, } 570 \mathrm{~m}\end{array}$ & MN733398 & A.Kaplan $D U F 88$ \\
\hline 3 & $\begin{array}{l}\text { V. villosa Roth subsp. varia } \\
\text { (Host) Corb. }\end{array}$ & Cracca & $\begin{array}{l}\text { Adiyaman, Bozova to Adiyaman, } \\
18.5 \mathrm{~km}, 589 \mathrm{~m}\end{array}$ & MN733439 & A.Kaplan $D U F 48$ \\
\hline 4 & V. mollis Boiss. \& Hausskn. & Hypechusa & $\begin{array}{l}\text { Adiyaman, Hilvan to Bozova, } \\
\text { Külünçe village, } 5 \mathrm{~km} .600 \mathrm{~m}\end{array}$ & MN733450 & A.Kaplan $D U F 40$ \\
\hline 5 & V. galeata Boiss. & Hypechusa & $\begin{array}{l}\text { Adiyaman, Hilvan to Bozova, } \\
\text { Külünçe village, } 5 \mathrm{~km}, 600 \mathrm{~m}\end{array}$ & MN736208 & A.Kaplan DUF 44 \\
\hline 6 & V. sativa L. subsp. sativa & Vicia & $\begin{array}{l}\text { Diyarbakır, Dicle University } \\
\text { campus, Bakbako stream around, } \\
630-640 \mathrm{~m}\end{array}$ & MN736336 & A.Kaplan $D U F 82$ \\
\hline 7 & $\begin{array}{l}\text { V. sativa L.subsp. nigra } \\
\text { L.(Ehrh.) var. nigra L.(Ehrh.) }\end{array}$ & Vicia & $\begin{array}{l}\text { Diyarbakır, Çermik to Çüngüş, } \\
6-7 \mathrm{~km}, 703 \mathrm{~m}\end{array}$ & MN738731 & $\begin{array}{c}\text { A.Kaplan\&A.S.Ertekin } \\
\text { DUF } 35\end{array}$ \\
\hline 8 & $\begin{array}{l}\text { V. michauxii } \\
\text { Sprengel.var.stenophylla } \\
\text { Boiss. }\end{array}$ & Peregrinae & $\begin{array}{l}\text { Şanlıurfa, Şanliurfa to Suruç, } \\
\text { 18km,666m. }\end{array}$ & MN736412 & A.Kaplan $D U F 08$ \\
\hline 9 & $\begin{array}{l}\text { V. monantha } \text { Retz } \\
\text { subsp.monantha Retz. }\end{array}$ & Cracca & Şanlıurfa, Akçakale,382m. & MN741043 & A.Kaplan DUF 09 \\
\hline 10 & V. anatolica Turril. & Hypechusa & $\begin{array}{l}\text { Mardin, Exit of Mardin } 2 \\
\mathrm{~km}, 1095 \mathrm{~m} .\end{array}$ & MN736415 & A.Kaplan $D U F 12$ \\
\hline 11 & V. peregrina $\mathrm{L}$. & Peregrinae & $\begin{array}{l}\text { Siirt, Çatılı village, } \\
1475 \mathrm{~m}\end{array}$ & MN736414 & A.Kaplan $D U F 73$ \\
\hline 12 & $\begin{array}{l}\text { V. noeana Reuter ex Boiss } \\
\text { var. noeana Reuter ex Boiss. }\end{array}$ & Hypechusa & $\begin{array}{l}\text { Diyarbakır, Ergani to Çermik, } \\
13 \mathrm{~km}, 870-875 \mathrm{~m}\end{array}$ & MN741159 & $\begin{array}{c}\text { A.Kaplan\&A.S.Ertekin } \\
\text { DUF } 16\end{array}$ \\
\hline 13 & $\begin{array}{l}\text { V. sericocarpa Fenzl. var. } \\
\text { sericocarpa Fenzl. }\end{array}$ & Hypechusa & $\begin{array}{l}\text { Diyarbakır, Ergani to Çermik } \\
13 \mathrm{~km}, 870-875 \mathrm{~m}\end{array}$ & MN741118 & $\begin{array}{c}\text { A.Kaplan\&A.S.Ertekin } \\
\text { DUF } 18\end{array}$ \\
\hline 14 & V. lathyroides $\mathrm{L}$. & Lathyroides & $\begin{array}{l}\text { Diyarbakır, Çermik to Çüngüş, } \\
11.5-12 \mathrm{~km}, 905 \mathrm{~m}\end{array}$ & MN736416 & $\begin{array}{c}\text { A.Kaplan\&A.S.Ertekin } \\
\text { DUF } 39\end{array}$ \\
\hline 15 & V. ervilia (L.) Willd. & Ervilia & $\begin{array}{l}\text { Diyarbakır, Ergani to Çermik, } \\
\text { 16- } 17 \mathrm{~km}, 880 \mathrm{~m}\end{array}$ & MN736417 & $\begin{array}{c}\text { A.Kaplan\&A.S.Ertekin } \\
\text { DUF } 21\end{array}$ \\
\hline 16 & V. galilaea Plitm.Et. Zoh. & Narbonensis & $\begin{array}{l}\text { Diyarbakır, Çermik to Siverek, } \\
1 \mathrm{~km}, 732 \mathrm{~m}\end{array}$ & MN736418 & $\begin{array}{c}\text { A.Kaplan\&A.S.Ertekin } \\
\text { DUF } 32\end{array}$ \\
\hline 17 & $\begin{array}{l}\text { V. cracca L. subsp. } \\
\text { Stenophylla } \text { Vel. }\end{array}$ & Cracca & $\begin{array}{l}\text { Adiyaman, Ferryboat to Siverek, } \\
4 \mathrm{~km}, 588 \mathrm{~m}\end{array}$ & MN741044 & A.Kaplan DUF 56 \\
\hline 18 & V. palaestina Boiss. & Cracca & $\begin{array}{l}\text { Diyarbakır, Çermik to Çüngüş } \\
11.5-12 \mathrm{~km}, 905 \mathrm{~m}\end{array}$ & MN736419 & $\begin{array}{c}\text { A.Kaplan\&A.S.Ertekin } \\
\text { DUF } 38\end{array}$ \\
\hline 19 & $\begin{array}{l}\text { V. lutea var. hirta (Balbis) } \\
\text { Lois. }\end{array}$ & Hypechusa & $\begin{array}{l}\text { Diyarbakır, Devegeçidi picnic } \\
\text { area, } 732 \mathrm{~m} \text {. }\end{array}$ & MN736420 & $\begin{array}{c}\text { A.Kaplan\&A.S.Ertekin } \\
\text { DUF } 59\end{array}$ \\
\hline 20 & $\begin{array}{l}\text { V. esdraelonensis Warb. \& } \\
\text { Eig }\end{array}$ & Hypechusa & $\begin{array}{l}\text { Diyarbakır, the way of } \\
\text { Bilgekışla, } 3.5 \mathrm{~km}, 740 \mathrm{~m}\end{array}$ & MN736422 & $\begin{array}{c}\text { A.Kaplan\&A.S.Ertekin } \\
\text { DUF } 60\end{array}$ \\
\hline 21 & $\begin{array}{l}\text { V. villosa } \text { Roth subsp. } \\
\text { Eriocarpa (Hausskn) P.Ball. }\end{array}$ & Cracca & $\begin{array}{l}\text { Adiyaman, Hilvan to Bozova, } \\
\text { Külünçe village, } 5 \mathrm{~km}, 600 \mathrm{~m}\end{array}$ & MN736423 & A.Kaplan DUF 41 \\
\hline 22 & $\begin{array}{l}\text { V. sativa } \text { L. subsp. } \\
\text { Amphicarpa (Dorth.) } \\
\text { Aschers. \& Graebn. }\end{array}$ & Vicia & $\begin{array}{l}\text { Diyarbakır, Dicle University } \\
\text { campus, 630-640m }\end{array}$ & MN736424 & A.Kaplan $D U F 80$ \\
\hline 23 & V. assyriaca Boiss. & Hypechusa & $\begin{array}{l}\text { Şanlıurfa, Siverek Avurtepe } \\
\text { village, Otlu avenue, } 1080 \mathrm{~m}\end{array}$ & MN736425 & A.S.Ertekin DUF 79 \\
\hline 24 & $\begin{array}{l}\text { V. montbretii Fisch. \& } \\
\text { C.A.Mey. }\end{array}$ & Lentopsis & $\begin{array}{l}\text { Şanliurfa, Siverek Avurtepe } \\
\text { village, Otlu avenue, } 1080 \mathrm{~m}\end{array}$ & MN736426 & A.S.Ertekin $D U F 78$ \\
\hline 25 & V. caesarea Boiss.\& Bal. & Lentopsis & $\begin{array}{l}\text { Siirt, Şirvan to Madenköy, } \\
4-5 \mathrm{~km}, 1020 \mathrm{~m}\end{array}$ & MN736432 & A.S.Ertekin DUF 184 \\
\hline 26 & Lathyrus cassius Boiss. & Lathyrus & $\begin{array}{l}\text { Diyarbakır, the way of } \\
\text { Bilgekışla, } 3.5 \mathrm{~km}, 740 \mathrm{~m}\end{array}$ & MN736435 & A.S.Ertekin DUF 4241 \\
\hline 27 & Lathyrus inconspicuous L. & Linearicarpus & $\begin{array}{l}\text { Gaziantep, Islahiye Bahçe to } \\
\text { Gaziantep, } 22 \mathrm{~km}\end{array}$ & MN738502 & A.S.Ertekin DUF 5249 \\
\hline
\end{tabular}




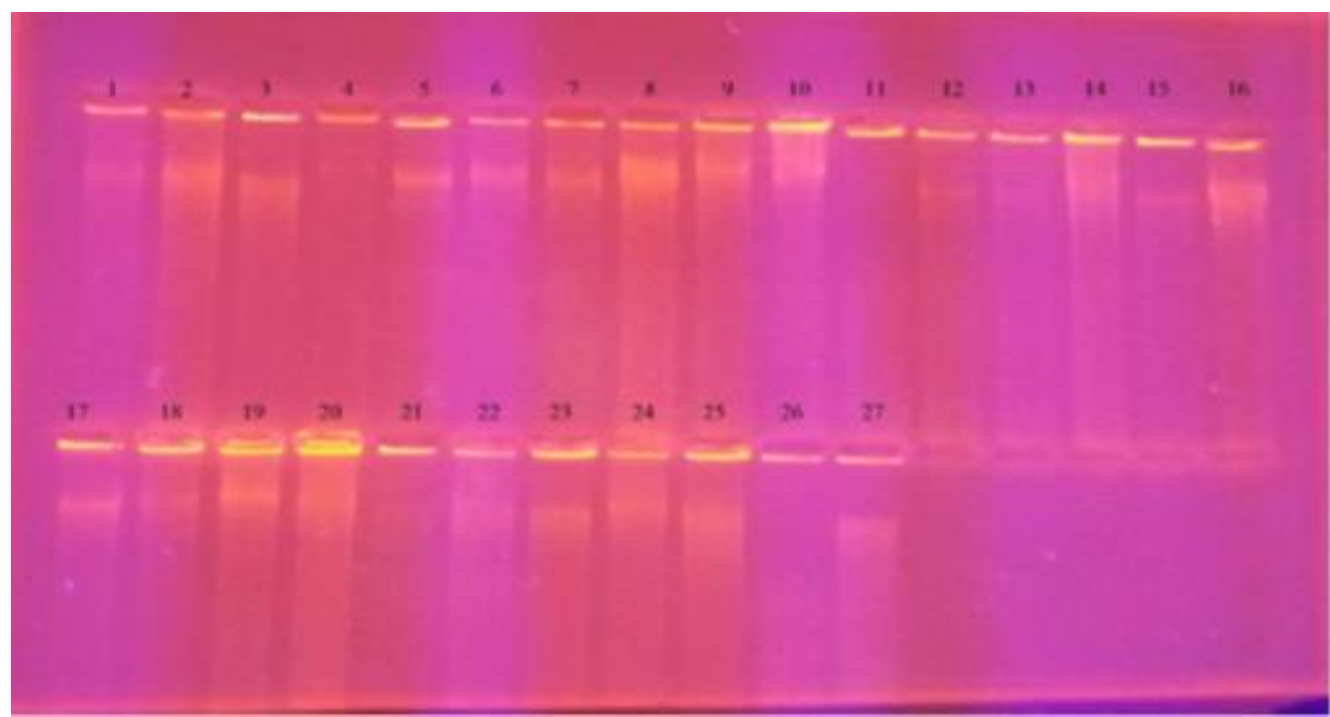

Figure $1.1 \%$ gel image of Vicia taxa genomic DNA samples

1) V. narbonensis var. narbonensis 2) V. hybrida 3) V. villosa subsp. varia 4) V. mollis 5) V. galeata 6) V. sativa subsp. sativa 7) $V$. sativa $\mathrm{L}$. subsp. nigra var. nigra 8)V. michauxii var. stenophylla 9) V. monantha subsp. monantha 10) V. anatolica 11) V. peregrina 12) V. noeana var. noeana 13) V. sericocarpa var. sericocarpa 14) V. lathyroides 15) V. ervilia 16) V. galilaea 17) V. cracca subsp. stenophylla 18) V. palaestina 19) V. lutea var. hirta 20) V. esdraelonensis 21) V. villosa subsp. eriocarpa 22) V. sativa subsp. amphicarpa 23) V. assyriaca 24) V. montbretii 25) V. caesarea 26) Lathyrus cassius 27) Lathyrus inconspicuous

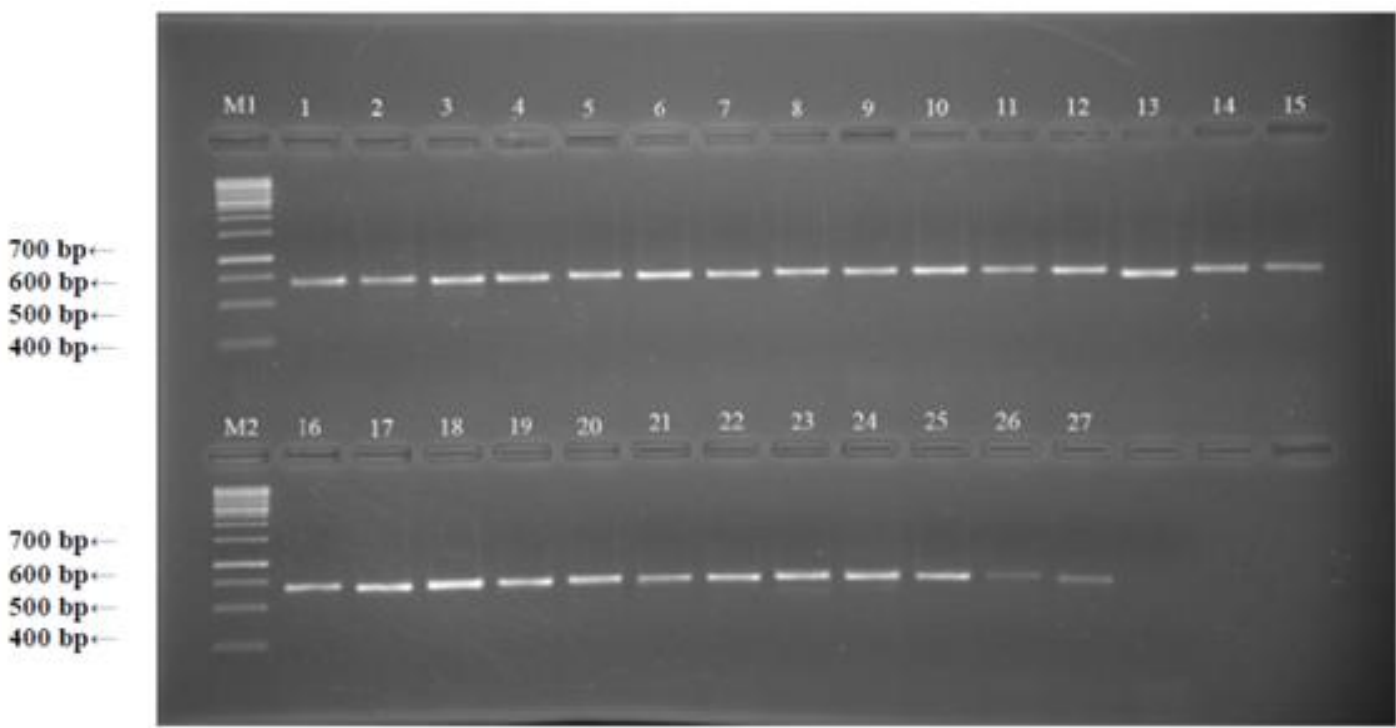

Figure 2. 2\% agarose gel image of PCR products of Vicia taxa belonging to ITS region

1) V. narbonensis var. narbonensis 2) V. hybrida 3) V. villosa subsp. varia 4) V. mollis 5) V. galeata 6) V.sativa subsp. sativa 7) V. sativa $\mathrm{L}$. subsp. nigra var. nigra 8) V. michauxii var. stenophylla 9) V. monantha subsp. monantha 10) V. anatolica 11) V. peregrina 12) V. noeana var. noeana 13) V. sericocarpa var. sericocarpa 14) V. lathyroides 15) V. ervilia 16) V. galilaea 17) V. cracca subsp. stenophylla 18) V. palaestina 19) V. lutea var. hirta 20) V. esdraelonensis 21) V. villosa subsp. eriocarpa 22) V. sativa subsp. amphicarpa 23) V. assyriaca 24) V. montbretii 25) V. caesarea 26) Lathyrus cassius 27) Lathyrus inconspicuous

\section{Sequence Alignment and Phylognetic Analysis}

Total nucleotide length (base pair, bp) (Table 2), distance matrix among Vicia species were determined using Molecular Evolutionary Genetics Analysis software (MEGA 5.1; [Beta 2] (Table 3). DNA sequences were aligned using the Clustal $\mathrm{W}$ program. The sequence data was analyzed using the UPGMA method (Figure 3) based on the Kimura model based on the Kimura model (Kimura 1980) and bootstrap analysis with 1000 replications (Felsenstein, 1985). According to the Kress et al. (2005) bootstrap support values, $>85 \%$ is defined as very strong, $70-85 \%$ as strong, $50-70 \%$ as weak and $<50 \%$ as very weak. A bootstrap support of $70 \%$ or greater is often associated with the identification of the correct phylogeny. If the bootstrap support for a certain branch is below 50\% it is concluded that the relationship between the species is not fully clarified and the branching model is not determined. As a result, this branch will be determined in the tree as a fork (polytomy = uncertainty point) rather than a single node (Freeman and Herron, 1999).

\section{Results and Discussion}

Molecular methods have been used as an important tool in recent years in classifying plants and understanding their evolution (Soltis et al., 1992). Classification studies of angiosperms at the level of genus and species have focused on using ITS sequence analysis studies. For this reason, ITS sequences have been a valuable method in new phylogenetic organizations of angiosperms. 
Table 2. ITS region sizes in Vicia L. and Lathyrus L. (outgrup) taxa

\begin{tabular}{|c|c|c|c|c|c|}
\hline Taxon names & $\mathrm{T}(\mathrm{U})$ & $\mathrm{C}$ & A & $\mathrm{G}$ & Total \\
\hline Vicia narbonensis var. narbonensis & 26.9 & 23.9 & 22.3 & 26.9 & 624.0 \\
\hline Vicia hybrida & 29.2 & 22.8 & 22.4 & 25.6 & 504.0 \\
\hline Vicia villosa subsp. varia & 26.7 & 24.0 & 22.2 & 27.0 & 603.0 \\
\hline Vicia mollis & 29.9 & 21.9 & 22.1 & 26.1 & 479.0 \\
\hline Vicia galeata & 27.4 & 23.2 & 23.0 & 26.3 & 638.0 \\
\hline Vicia sativa subsp. sativa & 27.7 & 23.7 & 22.1 & 26.5 & 620.0 \\
\hline Vicia sativa subsp. nigra var. nigra & 27.5 & 23.8 & 22.3 & 26.4 & 622.0 \\
\hline Vicia michauxii var. stenophylla & 27.7 & 23.6 & 22.0 & 26.7 & 632.0 \\
\hline Vicia monantha subsp. monantha & 27.0 & 24.1 & 22.2 & 26.7 & 626.0 \\
\hline Vicia anatolica & 28.5 & 22.2 & 22.8 & 26.5 & 635.0 \\
\hline Vicia peregrina & 27.5 & 23.7 & 22.0 & 26.7 & 632.0 \\
\hline Vicia noeana var. noeana & 27.9 & 23.1 & 22.2 & 26.8 & 631.0 \\
\hline Vicia sericocarpa var. sericocarpa & 27.8 & 23.0 & 22.6 & 26.6 & 625.0 \\
\hline Vicia lathyroides & 27.1 & 23.7 & 21.8 & 27.3 & 641.0 \\
\hline Vicia ervilia & 28.5 & 22.2 & 22.3 & 27.0 & 641.0 \\
\hline Vicia galilaea & 26.6 & 24.3 & 22.0 & 27.1 & 672.0 \\
\hline Vicia cracca subsp. stenophylla & 26.9 & 23.8 & 22.4 & 26.9 & 621.0 \\
\hline Vicia palaestina & 27.3 & 23.8 & 22.2 & 26.7 & 630.0 \\
\hline Vicia lutea var. hirta & 27.3 & 24.0 & 21.7 & 27.0 & 641.0 \\
\hline Vicia esdraelonensis & 27.5 & 23.5 & 22.5 & 26.4 & 639.0 \\
\hline Vicia villosa subsp. eriocarpa & 26.7 & 24.1 & 22.4 & 26.8 & 615.0 \\
\hline Vicia sativa subsp. amphicarpa & 27.6 & 24.0 & 21.6 & 26.7 & 633.0 \\
\hline Vicia assyriaca & 28.1 & 23.0 & 22.6 & 26.3 & 634.0 \\
\hline Vicia montbretii & 28.1 & 23.9 & 21.1 & 26.9 & 587.0 \\
\hline Vicia caesarea & 28.1 & 23.5 & 21.0 & 27.4 & 620.0 \\
\hline Lathyrus cassius & 26.9 & 24.3 & 22.0 & 26.9 & 674.0 \\
\hline Lathyrus inconspicuous & 27.8 & 23.0 & 22.4 & 26.8 & 634.0 \\
\hline Avg. & 27.6 & 23.5 & 22.2 & 26.7 & 620.5 \\
\hline
\end{tabular}

Table 3. Distance matrix among Vicia L. and Lathyrus L. (outgrup) taxa

\begin{tabular}{|c|c|c|c|c|c|c|c|c|c|c|c|c|c|}
\hline & 1 & 2 & 3 & 4 & 5 & 6 & 7 & 8 & 9 & 10 & 11 & 12 & 13 \\
\hline \multicolumn{14}{|l|}{ Vicia narbonensis var. narbonensis } \\
\hline Vicia hybrida & 0.025 & & & & & & & & & & & & \\
\hline Vicia villosa subsp. varia & 0.032 & 0.032 & & & & & & & & & & & \\
\hline Vicia mollis & 0.030 & 0.018 & 0.032 & & & & & & & & & & \\
\hline Vicia galeata & 0.032 & 0.035 & 0.034 & 0.030 & & & & & & & & & \\
\hline Vicia sativa subsp. sativa & 0.035 & 0.032 & 0.037 & 0.028 & 0.035 & & & & & & & & \\
\hline Vicia sativa subsp. nigra var. nigra & 0.030 & 0.028 & 0.032 & 0.023 & 0.030 & 0.005 & & & & & & & \\
\hline Vicia michauxii var. stenophylla & 0.023 & 0.016 & 0.025 & 0.016 & 0.021 & 0.025 & 0.021 & & & & & & \\
\hline Vicia monantha subsp. monantha & 0.028 & 0.027 & 0.018 & 0.028 & 0.032 & 0.028 & 0.023 & 0.021 & & & & & \\
\hline Vicia anatolica & 0.037 & 0.035 & 0.035 & 0.021 & 0.032 & 0.039 & 0.035 & 0.023 & 0.035 & & & & \\
\hline Vicia peregrina & 0.025 & 0.018 & 0.027 & 0.018 & 0.023 & 0.028 & 0.023 & 0.002 & 0.023 & 0.025 & & & \\
\hline Vicia noeana var. noeana & 0.030 & 0.028 & 0.030 & 0.023 & 0.016 & 0.028 & 0.023 & 0.016 & 0.025 & 0.030 & 0.018 & & \\
\hline Vicia sericocarpa var. sericocarpa & 0.035 & 0.028 & 0.037 & 0.023 & 0.039 & 0.037 & 0.032 & 0.025 & 0.032 & 0.030 & 0.028 & 0.032 & \\
\hline Vicia lathyroides & 0.025 & 0.023 & 0.027 & 0.023 & 0.030 & 0.023 & 0.018 & 0.016 & 0.018 & 0.030 & 0.018 & 0.023 & 0.028 \\
\hline Vicia ervilia & 0.039 & 0.037 & 0.039 & 0.030 & 0.042 & 0.039 & 0.035 & 0.028 & 0.039 & 0.042 & 0.030 & 0.035 & 0.044 \\
\hline Vicia galilaea & 0.007 & 0.018 & 0.025 & 0.023 & 0.025 & 0.028 & 0.023 & 0.016 & 0.021 & 0.030 & 0.018 & 0.023 & 0.028 \\
\hline Vicia cracca subsp. stenophylla & 0.030 & 0.030 & 0.014 & 0.025 & 0.030 & 0.035 & 0.030 & 0.018 & 0.016 & 0.032 & 0.021 & 0.023 & 0.035 \\
\hline Vicia palaestina & 0.030 & 0.030 & 0.025 & 0.025 & 0.030 & 0.035 & 0.030 & 0.018 & 0.021 & 0.032 & 0.021 & 0.023 & 0.035 \\
\hline Vicia lutea var. hirta & 0.025 & 0.023 & 0.025 & 0.023 & 0.025 & 0.028 & 0.023 & 0.016 & 0.021 & 0.030 & 0.018 & 0.018 & 0.028 \\
\hline Vicia esdraelonensis & 0.030 & 0.028 & 0.030 & 0.023 & 0.016 & 0.028 & 0.023 & 0.016 & 0.025 & 0.030 & 0.018 & 0.005 & 0.032 \\
\hline Vicia villosa subsp. eriocarpa & 0.032 & 0.032 & 0.005 & 0.032 & 0.034 & 0.037 & 0.032 & 0.025 & 0.018 & 0.039 & 0.027 & 0.030 & 0.037 \\
\hline Vicia sativa subsp. amphicarpa & 0.032 & 0.030 & 0.039 & 0.030 & 0.032 & 0.011 & 0.007 & 0.028 & 0.030 & 0.0042 & 0.030 & 0.025 & 0.040 \\
\hline Vicia assyriaca & 0.030 & 0.028 & 0.030 & 0.023 & 0.016 & 0.028 & 0.023 & 0.016 & 0.025 & 0.030 & 0.018 & 0.005 & 0.032 \\
\hline Vicia montbretii & 0.027 & 0.027 & 0.018 & 0.027 & 0.025 & 0.032 & 0.028 & 0.016 & 0.018 & 0.030 & 0.018 & 0.025 & 0.032 \\
\hline Vicia caesarea & 0.032 & 0.032 & 0.027 & 0.027 & 0.037 & 0.032 & 0.027 & 0.025 & 0.023 & 0.039 & 0.027 & 0.030 & 0.037 \\
\hline Lathyrus cassius & 0.047 & 0.044 & 0.049 & 0.044 & 0.049 & 0.044 & 0.039 & 0.032 & 0.044 & 0.051 & 0.035 & 0.044 & 0.039 \\
\hline \multirow{2}{*}{ Lathyrus inconspicuous } & 0.054 & 0.046 & 0.056 & 0.046 & 0.054 & 0.051 & 0.046 & 0.039 & 0.051 & 0.054 & 0.042 & 0.046 & 0.049 \\
\hline & 14 & 15 & 16 & 17 & 18 & 19 & 20 & 21 & 22 & 23 & 24 & 25 & 26 \\
\hline Vicia e & 0.035 & & & & & & & & & & & & \\
\hline Vicia galilaea & 0.018 & 0.035 & & & & & & & & & & & \\
\hline Vicia cracca subsp. stenophylla & 0.025 & 0.037 & 0.023 & & & & & & & & & & \\
\hline Vicia palaestina & 0.025 & 0.037 & 0.023 & 0.018 & & & & & & & & & \\
\hline Vicia lutea var. hirta & 0.018 & 0.035 & 0.018 & 0.023 & 0.023 & & & & & & & & \\
\hline Vicia esdraelonensis & 0.023 & 0.035 & 0.023 & 0.023 & 0.023 & 0.018 & & & & & & & \\
\hline Vicia villosa subsp. eriocarpa & 0.027 & 0.039 & 0.025 & 0.014 & 0.025 & 0.025 & 0.030 & & & & & & \\
\hline Vicia sativa subsp. amphicarpa & 0.025 & 0.042 & 0.025 & 0.037 & 0.037 & 0.030 & 0.025 & 0.039 & & & & & \\
\hline Vicia assyriaca & 0.023 & 0.035 & 0.023 & 0.023 & 0.023 & 0.018 & 0.005 & 0.027 & 0.025 & & & & \\
\hline Vicia montbretii & 0.023 & 0.039 & 0.021 & 0.016 & 0.021 & 0.021 & 0.025 & 0.018 & 0.035 & 0.025 & & & \\
\hline Vicia caesarea & 0.025 & 0.035 & 0.025 & 0.025 & 0.025 & 0.025 & 0.030 & 0.027 & 0.035 & 0.030 & 0.023 & & \\
\hline Lathyrus cassius & 0.039 & 0.056 & 0.039 & 0.044 & 0.046 & 0.035 & 0.044 & 0.049 & 0.047 & 0.044 & 0.039 & 0.044 & \\
\hline Lathyrus inconspicuous & 0.046 & 0.059 & 0.046 & 0.049 & 0.049 & 0.046 & 0.046 & 0.056 & 0.054 & 0.046 & 0.051 & 0.051 & 0.037 \\
\hline
\end{tabular}




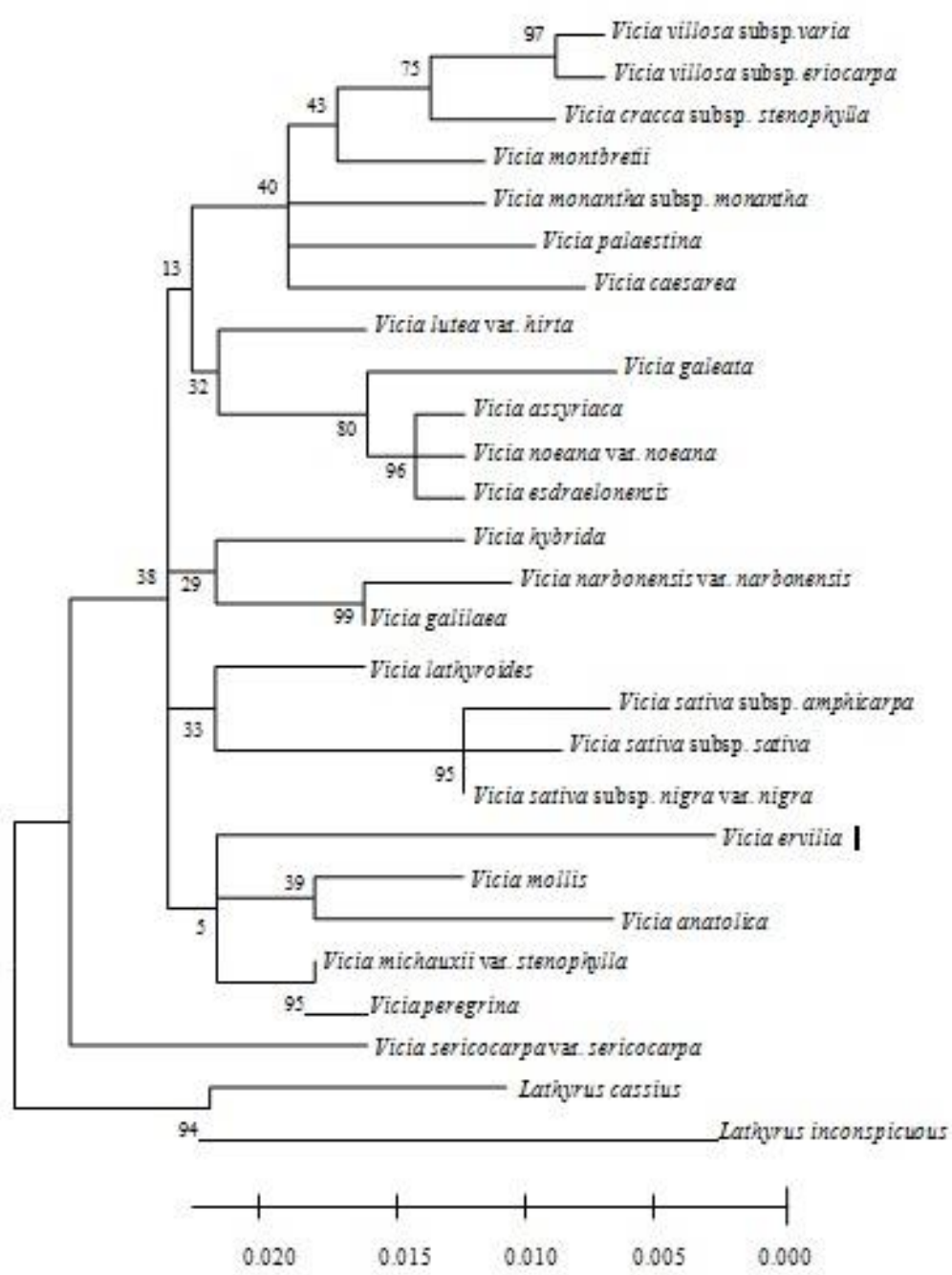

Figure 3. Phylogenetic tree of Vicia taxa based on (UPGMA) analysis of the ITS region. Lathyrus cassius and Lathyrus insconspicuous were used as a outgroup. Bootstrap analysis of UPGMA was based on 1000 replicates.

Percentage of each branch shows the support rate of that branch (Bootstrap value)

The data obtained as a result of the analysis of ITS base sequences make important contributions to the solution of problems existing in taxonomic categories. Generally, by looking at the ITS variations of taxa, the attempt can be made to identify relatives of the related taxa (Baldwin et al., 1995).

As the first parameter that constitutes the first step of the study, PCR was performed on gDNA samples of targeted species, the presence of bands was visualized using 1\% agarose gel electrophoresis (Figure 1). Amplicons (ITS region) were then visualized by electrophoresis on $2 \%$ agarose gel (Figure 2).

The base ratios obtained from approximately $620 \mathrm{bp}$ of ITS genes analyzed in the series are given in Table 2 . When Table 2 is examined, the sizes of the ITS regions can be seen to vary from $479 \mathrm{bp}$ (Vicia mollis) to $672 \mathrm{bp}$ in Vicia galilaea. In order of magnitude this appears to be: A: $22.2 \%$, G: $26.7 \%$, C: $23.5 \%$, T (U):27.6\%. DNA sequences related to the samples have a $\mathrm{G}-\mathrm{C}$ ratio $(50.2 \%)$ and an $\mathrm{A}$ - $T$ ratio $(49.8 \%)$ and the values are close to each other. The distance between the samples obtained using the MEGA program and the Kimura 2-Parameter base change model is given in Table 3. When Table 3 is examined, the smallest genetic distance value (0.005) was observed between 21-3, 7-6 and 20-12, while the largest genetic distance value
(0.044) was observed between samples 15 and 13. These values are genetically significant in the genus and this shows that there are no differences.

The phylogenetic results from ITS region sequences were found to be generally useful for understanding the evolutionary relationships of species and sections of Vicia. In the unweighted pair group method with arithmetic mean (UPGMA) tree obtained in the present study (Figure 3), it was observed that the Lathyrus species used as an outgroup were the first to be separated and this branch was supported with a bootstrap value of $94 \%$. The ingroup consists of two main clades. The first clade is divided into five subclades, consisting of the first subclade: V. villosa subsp. varia, $V$. villosa subsp. eriocarpa, V. cracca subsp. stenophylla, $V$. monantha subsp. monantha, V. montbretii, V. caesarea, and $V$. palaestina; the second subclade: V. lutea var. hirta, $V$. galeata, V. assyriaca, V. esdraelonensis, and V. noean var. noeana; the third subclade: $V$. hybrida, $V$. narbonensis var. narbonensis, and $V$. galilaea; the fourth subclade: V.sativa subsp. sativa, V. sativa subsp. nigra var. nigra, V. sativa. subsp. amphicarpa, and $V$. lathyroides and the fifth subclade, V. mollis, V. michauxii var. stenophylla, $V$. anatolica, V.peregrina, and V. ervilia. The second clade is $V$. sericocarpa var. sericocarpa. 
Using the data in Figure 3, the first subclade was found to consist of two sections of species. The first was sect. Cracca (V. villosa subsp. varia, V. villosa subsp. eriocarpa, $V$. cracca subsp. stenophylla, V. monantha subsp. monantha, and $V$. palaestina), and the second was sect. Lentopsis (V. montbretii, V. caesarea). The species belonging to sect. Cracca were in the same group. We can see that the reliability of the branch containing $V$. villosa subsp. eriocarpa and V. villosa subsp. varia is $97 \%$. V. cracca subsp. stenophylla was linked to this branch with a $75 \%$ reliable bootstrap value. Molecular data based on sequences of the ITS with high bootstrap values supports these species being placed in the same group. Other species belonging to the section are linked with weak bootstrap values. The species belonging to sect. Lentopsis are scattered within the group. Since the bootstrap values are as low as $43 \%$ and $40 \%$, there can be no clear interpretation about the relationship between these taxa. We can say that polytomy has been found since the reliability valuesare below $50 \%$. Therefore, the parser has not provides any information. Schaefer et al. (2012), in their study of sect. Lentopsis, examined the species in the same branch of the tree (maximum likelihood). The morphological data obtained in this study revealed that the variable characteristics of the peduncle length, the number of flowers per peduncle, flower sizes, the number of leaflet pairs, the leaflet shape, the fruit shape and the aspect ratio were the most common among these species. Leht (2009), examined the $V$. palaestina, V. villosa, and $V$. cracca taxa, which are among the Cracca section taxa, in the phylogenetic tree that he obtained in his study, and made a new cladistic analysis of 91 morphological data. In parallel with our work, Binzat (2012), Foladi et al. (2003), Schaefer et al. (2012) all examined sect. Cracca taxa within the same group in their studies on the molecular phylogeny of Vicia species.

The second subclade included $V$. lutea var. hirta, $V$. galeata, V. assyriaca, V. esdraelonensis, and V. noeana var. noeana. These species belong to the section Hypechusa. In this subclade, V. esdraelonensis, V. noeana var. noeana and $V$. assyriaca were supported with a bootstrap value of $96 \%$ reliability, while $V$. assyriaca connection to this group was supported with a bootstrap value of $80 \%$ reliability. However, V. lutea var. hirta connection to this group was supported with a bootstrap value of $32 \%$ reliability and it was not informative. Molecular data based on sequences of the ITS with high bootstrap values support placing these species in this section. Maxted and Douglas (1997) found that in sect. Hypechusa the peduncle length can be divided into two series according to the shape and size of the corolla, the hairiness of the flag and the strength of the wings at the basal. These series are Hypechusa and Hyrcanicae. V. assyriaca, V. galeata, V. esdraelonensis, V. noeana var. noeana are included in the Hyrcanicae series. This information was confirmed in our tree through the supporting branches with high bootstrap values. Castiglione et al. (2007), in their cytological and molecular characterization (ITS region located in nrDNA) studies of $V$. esdraelonensis, found that the species belongs strictly in the section of Hypechusa, and that it is closely related to $V$. noeana. This finding coincides with those in our study. Schaefer et al. (2012), in their molecular phylogeny studies, determined that taxa belonging to the section, such as $V$. hyrcanica, $V$. noeana, $V$. galeata, $V$. lutea, $V$. esdraelonensis, were included in the same group. They reported that the Hypechusa section was not monophyletic. Caputo et al. (2006) also stated that the Hypechusa section is a heterogeneous section, not a monophyletic section, but noted that some species in the section are monophyletic. The phylogenetic tree we obtained supports this assertion.

The third subclade included $V$. hybrida (sect. Hypechusa), $V$. narbonensis var. narbonensis and $V$. galilaea (sect. Narbonensis). In our tree, the branch with $V$. narbonensis var. narbonensis and $V$. galilaea species was supported with a $99 \%$ bootstrap value. Molecular data based on sequences of the ITS with high bootstrap values support placing these species in this section. V. hybrida was connected to this branch with a bootstrap value of $29 \%$. Since this value was below 50\%, the branch collapsed and did not provide any exact information. In many studies (Enneking and Maxted, 1995; Fennel et al., 1998; Potokina, 1999; Schaefer et al., 2012), species belonging to sect.Narbonensis have been studied within the same branch. These results are in line with our study. Schaefer et al. (2012) reported that the section was monophyletic. Our study supports the finding that the section is monophyletic.

The fourth subclade included $V$. sativa subsp. sativa, $V$. sativa subsp. nigra var. nigra, $V$. sativa. subsp. amphicarpa (sect. Vicia), and V. lathyroides (sect. Lathyroides). The branch with $V$. sativa subsp. sativa, $V$. sativa subsp. nigra var. nigra, $V$. sativa. subsp. amphicarpa species was supported with a bootstrap value of $95 \%$ and this value indicates that a meaningful result has been achieved. Molecular data based on sequences of the ITS with high bootstrap support placing these species in this section. V. lathyroides was connected to this branch with a weak bootstrap value of $33 \%$. Since the bootstrap value of this branch was below $50 \%$, no clear information was provided and this branch collapsed. Van de Wouv et al. (2001) found that $V$. sativa subsp. amphicarpa and $V$. sativa subsp. nigra var. nigra are located close to each other, while V. sativa subsp. sativa is located farther away in the UPGMA phylogenetic tree they obtained. Potokina (1999) reported that the $V$. sativa complex coexists in the dendrograms they obtained in their study. Leht (2009), Potokina (1999) and Schaefer et al. (2012) reported that this section is not monophyletic but polyphyletic. However, Potokina et al. (1999) examined V. lathyroides species as a separate branch in their study and found $53 \%$ support for the branch where this species was located. They also found it close to the Vicia section in all the trees they created. Jaaska (2008) and Leht (2009) reported that they found the species to be close to the Vicia section. Although the bootstrap value of the $V$. lathyroides species was low, a close connection has been established with sect. Vicia in the studies mentioned above. Schaefer et al. (2012) determined that the section was not monophyletic. The results we obtained support those of the other recent studies.

The fifth subclade included $V$. mollis, $V$. anatolica (sect. Hypechusa) V. michauxii var. stenophylla, V. peregrina (sect. Peregrinae), and V. ervilia (sect. Ervilia). The branch with the highest bootstrap value in the group was the branch with $V$. michauxii var. stenophylla and $V$. 
peregrina, which was supported with a bootstrap value of 95\%. Molecular data based on sequences of the ITS with high bootstrap, support placing these species in this section. Many studies (Fennel et al., 1998; Potokina et al., 1999; Leht, 2009; Emre, 2011; Schaefer et al., 2012) found that these species belonged to the same branch and examined them in the Peregrinae section. Schaefer et al. (2012) found that the section was monophyletic. This result is in line with our study. Since the branch containing the $V$. mollis and $V$. anatolica species was only supported by a bootstrap value of $39 \%$, the branch collapsed and did not provide any discriminatory information. $V$. ervilia is located on a single, separate branch (monotypic clade) of the tree. Although it appears to be different from the other distributed species, there is no bootstrap support. Jaaska (2005) positioned the V. ervilia taxon in a separate branch on the tree in the dendrogram obtained in his study. $\mathrm{He}$ found that the group with which a taxon connection was formed was a group external to the other groups. Leht (2009) examined $V$. ervilia as a single-branch monotypic clade. According to Schaefer et al. (2012) dendograms obtained from the ITS region, $V$. ervilia belonged in sect. Ervilia as a monotypic clade. According to Binzat (2012), genus Vicia members have compound leaves. Most of them have a leaf ending with tendril. Only two of them have no tendrils. Instead of having tendrils, they have a mucro at the leaf's rachis ending (V.truncatula and V. ervilia). This property of the leaves is useful in infrageneric grouping. In terms of the monotypic nature of $V$. ervilia, we think that the information obtained in the ITS region may represent this morphological difference.

The second clade, V. sericocarpa var. sericocarpa, acted as the outer group of the second clade and has no bootstrap value. In the current study, this taxon was located separately. This is different from the findings of Schaefer et al. (2012), Leht (2009), and Binzat (2012). It has been generally studied within the Hypechusa section. However, El-Shanshoury and Soliman (1996) reported that $V$. sericocarpa was separate from the Hypechusa section and was located on the same branch as $V$. palaestina. According to the phylogenetic tree we obtained with the ITS region, this species has not yet been successfully parsed. Finally, the main branches formed in the phylogenetic tree obtained in the current study almost completely support the divisions into sections made according to the morphology of the genus.

In the field of taxonomy, the validity of hypotheses created with only morphological data may remain limited. We can say that the ITS region we used in our study is largely successful. The high genetic variation of the ITS region may be due to rapid concerted evolution and crossing over.

Consequently, a leguminous plant which Vicia L. a good source of nutrients that for animals, 14 to close the farming of its kind in Turkey, used as edible ( $V$. faba), are used as green manure (The amount of nitrogen detected by the vet per decare through its nodosity at the roots is $10-12$ kg.) as well as the same features a good time for the nectar of wild bees and honey bees (nectar) any research due to be held in Vicia sources will contribute to increasing the economic importance of this plant in Turkey. However, the fact that Vicia L. taxa, especially in some parts of Eastern Anatolia and Southeast Anatolia, is one of the third genetic differentiation regions in the world adds another importance to our study. It is important for the whole world to carefully conserve the gene resources of Vicia L. species, which are almost as old as human history, and many species are cultivated, belonging to the legume family. We hope that the economic integration studies, which are carried out at the end of the inventory and documentation studies in basic sciences all over the world, will now be carried out effectively in our country and that this information will serve this purpose.

\section{Acknowledgments}

The authors would like to thank David Duffy, for linguistically reviewing the paper.

\section{References}

Aceto S, Caputo P, Cozzolino S, Gaudio L. and Moretti A. 1999. Phylogeny and Evolution of Orchis and Allied Genera Based on ITS DNA Variation: Morphological Gaps and Molecular Continuity, Mol Phylo and Evol., 13 (1): 67-76.

Baldwin BG, Sanderson MJ, Porter JM, Wojciechowoski MF, Campell CS and Donoghue MJ. 1995. The ITS Region of Nuclear Ribosomal DNA: A Valuable Source of Evidence on Angiosperm Phylogeny, Annals of the Missouri Botanical Garden, 250-272.

Başbağ M, Hoşgören H, Aydın A. 2013. Vicia Taxa in the Flora of Turkey, Anadolu J. Agr. Sci., 28(1):59-66.

Binzat OK. 2012. Revision of Vicia L. (Leguminosae) in Central Anatolia, Turkey. Ph.D. Thesis, Middle East Technical University, Institute of Natural and Applied Sciences, September, Ankara.

Bryant JA, Hughes SG. 2011. Vicia, Chapter 14, C.kole (ed.). Wild Crop Relatives: Genomic and Breeding Resources, Legume Crops and Forages, Spriger verlag, Heidelberg.

Caputo P, Frediani M, Venora G, Ravalli C, Ambrosio M and Cremonini R. 2006. Nuclear DNA contents, rDNAs and karyotypes evolution in subgenus Vicia: III. Heterogeneous section Hypechusa, Protoplasma, 228: 167- 177.

Castiglione MR, Frediani M, Gelati MT, Ravalli C, Venora G, Caputo P and Cremonini R. 2007. Cytological and molecular characterization of Vicia esdraelonensis Warb. \& Eig: a rare taxon, Protoplasma, 231: 151- 159.

Davis PH. 1970. Vicia L. In: Flora of Turkey and the East Aegean Islands, Vol.3 (ed. Davis, P.H). p. 274-325. Edinburgh University Press, Edinburgh.

El-Shanshoury AR, Soliman SA. 1996. Electrophoretic Evidence for Subgeneric and Sectional Relationships of Some Species in Vicia L., Pak J Bot., 28: (2) 173-182.

Emre İ. 2011. Determination of Genetic Diversity in the Vicia L. (section Vicia) by Using SDS-PAGE, Pak J Bot., 43(3): 14291432.

Enneking D, Maxted N. 1995. Narbon bean (Vicia narbonensis L.). Evolution of Crop Plants, 2nd ed. Smartt, J., Simmonds, N. W., Longman: London, p. 316-321.

Felsenstein, J. 1985. Confidence limits on phylogenies: an approach using the bootstrap, Evolution., 39: 783- 791. 242.

Fennel SR, Powell W, Wright F, Ramsay G. and Waugh R. 1998. Phylogenetic relationships between Vicia faba (Fabaceae) and related species inferred from chloroplast $\operatorname{trn} L$ sequences, P1 Syst Evol., 212:247- 259.

Foladi FZ, Salimpour F, Sharifnia F, Ghanavati F. 2013. Phylogenetic study of tribe Vicieae based on Internal Transcribed Spacer (ITS), Ann of Biol Res., 4 (1):75-79.

Freeman S. and Herron JC. 1999. Evrimsel Analiz, Çıplak, B., Başıüyük. H.H., Karaytuğ,S. ve Gündüz. İ. (ed.), Palme Publishing., 28- 29, 438- 449. 
Guidetta R. and Michelozzi G. 1986. Biosystematic stu-dies on the Vicia villosa complex in Europe, Cando/lea., 41 (2): 399411.

Haider N, Nabulsi I, Kamary Y. 2010. Identification of species of Orchidaceae in Northern West of Syria based on chloroplast DNA, Russ J Genet., 46: 1067-1078.

Haider N, Nabulsi I, Miral1 N. 2012. Identification of species of Vicia subgenus Vicia (Fabaceae) using chloroplast DNA data, Turk J Agric For., 36: 1105-17.

Hanelt P. and Mettin D. 1989. Biosystematics of the genus Vicia L. (Leguminosae), Annu Rev Ecol Syst., 20: 199-223.

Heywood VH, Brummit RK, Culham A, Seberg O. 2007. Flowering Plant Families Of The World, Royal Botanic Gardens, Kew.

Hosseinzadeh Z, Pakravan M. and Tavassoli A. 2008. Micromorphology of Seed in Some Vicia Species from Iran, Rostaniha., 9(2): 97-107.

Jaaska V. 2005. Isozyme Variation and Phylogenetic Relationships in Vicia subgenus Cracca (Fabaceae), Ann of Bot., 96: 1085-1096.

Jaaska V. 2008. Isozymes Evidence on the Specific Distinctness and Phylogenetic Position of Vicia incisa (Fabaceae), Cent Europ J Biol., 3(2):169- 176.

Kahraman A, Binzat OK, Doğan M. 2013. Pollen morphology of some taxa of Vicia L. subgenus Vicia (Fabaceae) Turkey, Plant syst evol., 299: 1749-1760.

Kaplan A. 2014. The Morphological and Molecular Revision of the Genus Vicia L. (Fabaceae) Growing in the South Eastern Anatolia Region, Ph.D. Thesis, Dicle University, Institute of Natural And Applied Sciences,Diyarbakır.

Kimura MA. 1980. Simple Method for Estimating Evolutionary Rates of Base Substitutions Through Comparative Studies of Nucleotide Sequences, J Mol Evol., 16: 111-120.

Kress WJ, Liu AZ, Newman M, Li QJ. 2005. The molecular phylogeny of Alpinia (Zingiberaceae): a complex and polyphyletic genus of gingers, American J Bot., 92(1): 167178.

Kupicha FK. 1976. The infrageneric structure of Vicia L. Notes Roy Bot Garden Edinburgh, 34: 287-326.

Lefort F, Lally M, Thompson D, Douglas GC. 1998. Morphological traits, microsatellite fingerprinting and genetic relatedness of a stand of elite oaks $(Q$. robur L. $)$ at Tullynally, Ireland, Silvae Genetica, 47: 257-262.

Leht M. 2009. Phylogenetics of Vicia (Fabaceae) based on morphological data, Feddes Repertorium., 120 (7- 8): 379393.

LPWG, 2017. A new subfamily classification of the Leguminosae based on a taxonomically comprehensive phylogeny: The Legume Phylogeny Working Group (LPWG), Taxon, 66 :4477.
Maxted N, Douglas C. 1997. A phenetic investigation of Vicia section Hypechusa (Alef.) Asch. \& Graebner (Leguminosae, Papilionoideae, Vicieae), Lagascalia., 19(1- 2):345- 370.

Maxted N. 1995. An ecogeographical study of Vicia subgenus Vicia. Systematic and ecogeographic studies on crop genepools 8. International plant genetic Resources Institute. Rome. Italy.

Miller JT, Murphy DJ, Brown GK, Richardson DM, GonzálezOrozco CE. 2011. The evolution and phylogenetic placement of invasive Australian Acacia species. Diversity and Distributions (Diversity Distrib.)., 17: 848-860.

Navratilova A, Neumann P. and Macas J. 2003. Karyotype analyses of four Vicia species using in situ hybridization with repetetive sequnces, Ann of Bot., 91: 921-926,

Potokina EK, Tomooka N, Vaughan DA, Alexandrova T. and Xu RQ. 1999. Phylogeny of Vicia (Fabaceae) based on analaysis of RAPDs and RFLP of PCR amplified chloroplast genes, Gen Res and Crop Evol., 46: 149- 161.

Saqer MMF. 2014. Phylogenetic Relationship among Closely Related Species of the Genera Lens, Vicia, Lathyrus and Pisum (Leguminosae) in Palestine. MSc Thesis. An-Najah National University. Faculty of Graduate Studies. Palestine.

Schaefer H, Hechenleitner, P, Santos-Guerra A, Menezes de Sequeira M, Pennington TR, Kenicer G, Carine AM. 2012. Systematic, biogeography, and character evolution of the legume tribe Fabeae with special focus on the middle-Atlantic island lineages, BioMed Cent Evol Biol., 12: 250.

Simpson MG. 2012. Plant Systematics (2.edition ), Aytaç Z. (ed.), Nobel Publishing.

Soltis DE, Soltis PS, and Milligan BG. 1992. Intraspecific chloroplast DNA variation: Systematic and phylogenetic implications, p. 117-150, In P. S. Soltis et al. (ed.) Molecular systematic of plants, Chapman Hall. New York.

Tabur S, Civelek Ş and Bağcı E. 2002. Cytotaxonomic Studies on Some Vicia L. Species Growing in the Eastern Mediterranean and Southern Aegean Regions II, Acta Bot Hung., 44(1-2): 185- 204,

Van de Wouw M, Maxted N, Chabane K, Ford-Lloyd BV. 2001. Molecular Taxonomy of Vicia ser. Vicia Based on Amplified Fragment Lenght Polymorphisms, Plant Syst Evol., 229: 91105.

Vavilov N. 1994. Origin and Geography of Cultivated Crops. Cambridge Univ. Press. U. K.

Vavilov NI. 1950. The orgin, Variation, immunity and Breding of Cultivated Plants, Chronica Botanica.,13.

White TJ, Bruns T, Lee S, Taylor J. 1990. Amplification and direct sequencing of fungal ribosomal RNA genes for phylogenetics. PCR Protocols: A Guide to Methods and Applications, Innis, M., Gelfand, D., Sninsky, J., White, T. (ed.), Academic Press, San Diego, 315-322. 